\title{
Outer Membrane Vesicles of Helicobacter pylori TK I 402 are Involved in Biofilm Formation
}

\author{
Hideo Yonezawa*1, Takako Osaki ${ }^{1}$, Satoshi Kurata ${ }^{1}$, Minoru Fukuda ${ }^{2}$, \\ Hayato Kawakami ${ }^{3}$, Kuniyasu Ochiai ${ }^{4}$, Tomoko Hanawa ${ }^{1}$ and \\ Shigeru Kamiya ${ }^{1}$
}

Address: ${ }^{1}$ Department of Infectious Disease, Kyorin University School of Medicine, Shinkawa, Mitaka, Tokyo, 181-8611, Japan, ${ }^{2}$ Laboratory for Electron Microscopy, Kyorin University School of Medicine, Shinkawa, Mitaka, Tokyo, 181-8611, Japan, ${ }^{3}$ Department of Anatomy, Kyorin University School of Medicine, Shinkawa, Mitaka, Tokyo, 181-8611, Japan and ${ }^{4}$ Department of Bacteriology, Nihon University School of Dentistry, Kanda-Surugadai, Chiyoda-ku, Tokyo, 101-8310, Japan

Email: Hideo Yonezawa* - yonezawa@ks.kyorin-u.ac.jp; Takako Osaki - osaki@kyorin-u.ac.jp; Satoshi Kurata - kura@kyorin-u.ac.jp; Minoru Fukuda - kaze@kyorin-u.ac.jp; Hayato Kawakami - kaha@kyorin-u.ac.jp; Kuniyasu Ochiai - ochiai@dent.nihon-u.ac.jp; Tomoko Hanawa - thanawa@kyorin-u.ac.jp; Shigeru Kamiya - skamiya@ks.kyorin-u.ac.jp

* Corresponding author

Published: 15 September 2009

BMC Microbiology 2009, 9:197 doi:10.1186/147|-2180-9-197
Received: 23 March 2009

Accepted: 15 September 2009

This article is available from: http://www.biomedcentral.com//47/-2/80/9/197

(C) 2009 Yonezawa et al; licensee BioMed Central Ltd.

This is an Open Access article distributed under the terms of the Creative Commons Attribution License (http://creativecommons.org/licenses/by/2.0), which permits unrestricted use, distribution, and reproduction in any medium, provided the original work is properly cited.

\begin{abstract}
Background: Helicobacter pylori forms biofilms on glass surfaces at the air-liquid interface in in vitro batch cultures; however, biofilms of $H$. pylori have not been well characterized. In the present study, we analyzed the ability of $H$. pylori strains to form biofilms and characterized the underlying mechanisms of $H$. pylori biofilm formation.

Results: Strain TKI402 showed strong biofilm forming ability relative to the other strains in Brucella broth supplemented with 7\% FCS. The strong biofilm forming ability of TKI 402 is reflected the relative thickness of the biofilms. In addition, outer membrane vesicles (OMV) were detected within the matrix of only the TK 1402 biofilms. Biofilm formation was strongly correlated with the production of OMV in this strain. We further observed that strain TKI402 did not form thick biofilms in Brucella broth supplemented with $0.2 \% \beta$-cyclodextrin. However, the addition of the OMV-fraction collected from TKI 402 could enhance biofilm formation.
\end{abstract}

Conclusion: The results suggested that OMV produced from TKI402 play an important role in biofilm formation in strain TKI 402.

\section{Background}

Helicobacter pylori is a spiral, microaerophilic, noninvasive, gram-negative bacterium that colonizes the human gastrointestinal tract, primarily the stomach [1]. This organism has been identified as an aetiological agent of chronic active gastritis, peptic ulcer disease $[2,3]$, gastric adenocarcinoma [4], and mucosa-associated lymphoid tissue (MALT) lymphoma [5]. A number of factors such as the VacA cytotoxin, the cag pathogenicity island (cag PAI), motility, and the urease enzyme are known to be involved in the virulence of this organism [6-8].

Biofilm development is initiated when bacteria transit from a planktonic state to a lifestyle in which the microorganisms are firmly attached to biotic or abiotic surfaces, and biofilms are strongly implicated in bacterial virulence 
[9]. Biofilm formation is critical not only for environmental survival but also for successful infection by numerous pathogenic bacteria. Among human bacterial pathogens, the biofilms of Pseudomonas aeruginosa, Haemophilus influenzae, pathogenic Escherichia coli, Vibrio cholerae, staphylococci and streptococci are some of the best studied [1014]. Bacterial biofilms are frequently embedded in a selfproduced extracellular matrix [15]. The extracellular polymeric substance (EPS) matrix, which can constitute up to $90 \%$ of the biofilm biomass, is a complex mixture of exopolysaccharides, proteins, DNA and other macromolecules [16].

Previous studies have alluded to the ability of $H$. pylori to form biofilms $[17,18]$. A polysaccharide-containing biofilm has been observed at the air-liquid interface when $H$. pylori was grown in a glass fermenter [17]. H. pylori is also capable of binding to a heterotrophic mixed species biofilm grown on stainless steel coupons [18]. Recent studies indicated that 10 strains including some animal-adapted strains, clinical isolates and laboratory strains, were able to form similar three-dimensional architectures implicated in biofilm development $[19,20]$. Cellini et al. reported that an environmental $H$. pylori strain, named MDC1, displayed a well structured biofilm [19]. Cole et al. also indicated that mucin greatly accelerated planktonic growth relative to the expansion of $H$. pylori biofilms [2]. In addition, a recent study indicated that $H$. pylori can exist in human gastric mucosa forming biofilms [21]. These studies indicated that the topic of biofilm formation in this organism has the potential to contribute to our knowledge of $H$. pylori pathogenesis. However, little is known regarding the mechanism of $H$. pylori biofilm development. In the present study, we characterized the ability of 4 reference strains and 4 clinical isolates of $H$. pylori to form biofilms. Furthermore, we investigated the potential role of outer membrane vesicles (OMV) released from this organism in biofilm development.

\section{Results}

\section{Biofilm formation by $\mathbf{H}$. pylori strains}

We attempted to grow biofilms of the 8 strains of $H$. pylori on glass coverslip surfaces in Brucella broth supplemented with 7\% FCS with shaking for 3 days or 5 days and found that all strains formed biofilms at the liquid-gas interface of the cultures. Under these conditions, all of the strains except strain TK1402 formed relatively little biofilm biomass (Fig. 1A). In contrast, the clinically isolated strain TK1402 showed significantly higher levels of biofilm formation (Fig. 1A). The growth yields of these strains for 3or 5-days of culturing were comparable for all of the strains (Fig. 1B). To determine the kinetics of $H$. pylori biofilm formation, strains TK1402 and SS1 were assessed for biofilm forming ability and growth rates from day 1 to day 6 (Fig. 1C and 1D). Both strains showed similar growth kinetics with both strains fully grown within 2 days although the maximum titers of strain SS1 were slightly lower compared to that of strain TK1402. After 3 days of incubation, the growth yields were slightly decreased and plateaued at day 6 . On the other hand, biofilm formation by strain TK1402 increased until day 3 (Fig. 1C). After 3 days of incubation, biofilm formation reached a plateau up to day 6 . Biofilm formation by strain SS1 was not significantly different from day 1 to day 3 (Fig. 1D), and biofilm formation was significantly lower than that of TK1402 upon cultivation for up to 6 days.

\section{Morphological analysis of biofilms}

The biofilms were stained with a BacLight LIVE/DEAD bacterial viability kit solution which could differentiate between live cells (green) and dead cells (red). Strain TK1402 formed strong biofilms covering the entire visible area (Fig. 2A) but the other strain SS1 formed relatively poor biofilms (Fig. 2B). In the biofilms of both strains, the majority of the biofilm cells were stained green (Fig. 2A, and $2 \mathrm{~B}$ ). In order to confirm that the TK1402 biofilm cells were viable, the 2-day and 3-day biofilms cells were scrapped into PBS and the optical densities and the CFU values of the mixtures were evaluated (Table 1). The 2-day and 3-day cultures of this strain in Brucella broth supplemented with 7\% FCS were also measured as controls. The optical densities and CFU of the 3-day biofilm cells showed increases compared to those of 2-day biofilm cells. Further, the CFU values were normalized to optical densities. The values for the 2-day biofilm cells were similar compared to the controls (broth culture). However, the normalized values for 3-day biofilm cells tended to be decreased, although there was no significant difference in the normalized values, suggesting that 3 -day biofilm cells might contain some dead cells or morphologically altered cells.

Next we analyzed the biofilm thickness of strains TK1402, SS1, TK1029, and ATCC 49503 with CMLS observations. Strain TK1402 exhibited 2-fold or greater biofilm thickness compared to the other strains (Fig. 2C). To clarify the architectural characteristics of $H$. pylori biofilms, we compared TK1402 and SS1 biofilms by SEM analysis. In the biofilms of strain SS1, the bacteria attached to glass surfaces in thin layers (Fig. 2E). Interestingly, the biofilms consisted mainly of bleb-like or amorphous structures. On the other hand, the TK1402 biofilms were composed primarily of cells with bacillary morphology which were clearly outlined (Fig. 2D). In addition, these later bacteria showed layer formation with bacterial aggregates in the biofilms. The biofilm bacterial aggregates appeared to result from direct cell-cell attachment. Intriguingly, TK1402 biofilms showed the presence of many OMV-like structures on the glass surface as well as on the bacterial cell surfaces (Fig. 2D, white arrows). These structures were 
(A)

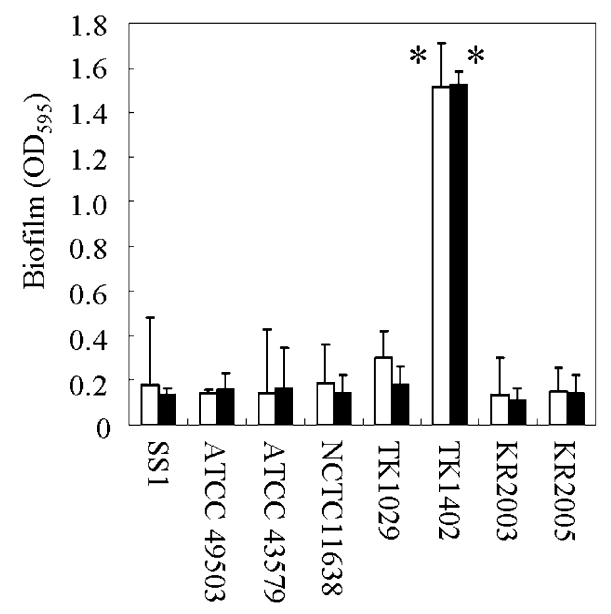

(C)

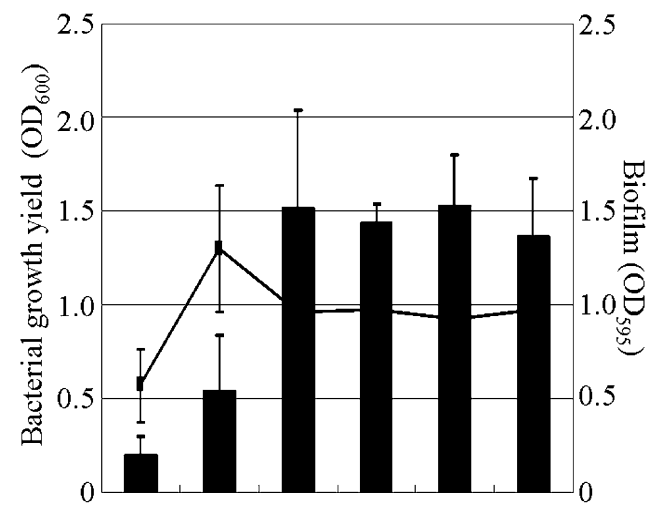

Day1 Day2 Day3 Day4 Day5 Day6
(B)

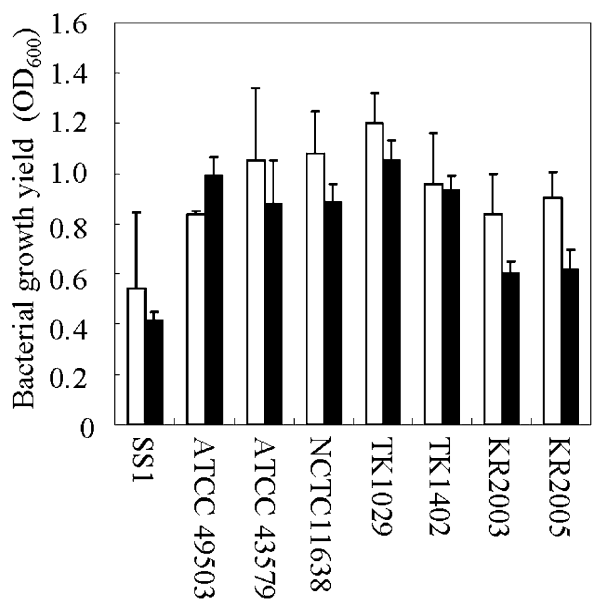

(D)

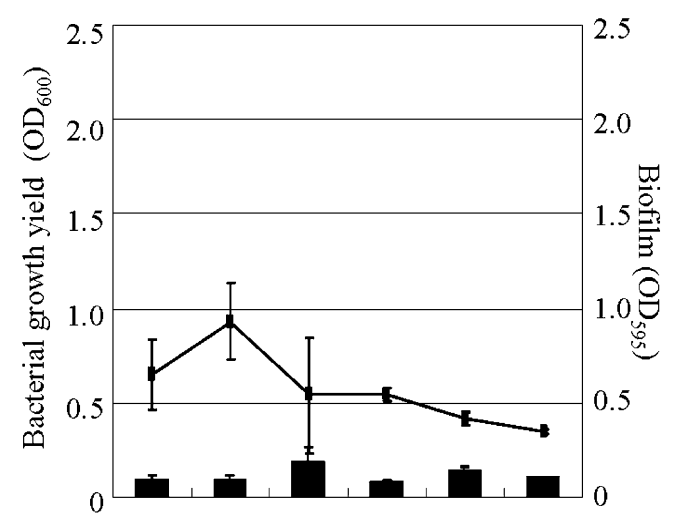

Day1 Day2 Day3 Day4 Day5 Day6

Figure I

(A) Biofilm formation by eight $\boldsymbol{H}$. pylori strains. The graph shows quantification of biofilms formed after 3-days (white bars) and 5-days (black bars) following culture in Brucella broth containing 7\% FCS. (B) Eight $H$. pylori strains were grown in Brucella broth containing $7 \%$ FCS-, and $\mathrm{OD}_{600}$ absorbance was measured at 3-days (white bars) and 5-days (black bars). (C, and D) Time course experiments for the H. pylori strains TKI402 (C) and SSI (D) biofilm formation and their growth. The biofilm mass of these strains are shown as black bars and the lines depict the $\mathrm{OD}_{600}$ absorbances of these strains. All of the results were expressed as the means \pm standard deviations from at least three independent experiments. *significantly different $(p<$ 0.05 ) relative level of biofilm formation (strain TKI 402 versus other strains).

not detected in the biofilms of the other strains (Fig. 2E and data not shown). A recent report indicated that OMV production from $H$. pylori clinical isolate MDC1 was apparent under SEM observation [19]. We thus decided to focus our attention more on the OMV-like structures in subsequent experiments.

\section{Potential role of the OMV in TKI402 biofilm formation}

We observed more closely the OMV-like structures in the thin-sectioned biofilms using TEM (Fig. 3). These struc- tures consisted primarily of bilayered proteolipids which were mainly spherical in shape (Fig. 3, black arrows). These structures also exhibited the characteristics typical of Gram negative bacterial OMV [22]. We confirmed that the OMV-fraction did not contain flagella by observation with SM and Western blotting with anti-flagella antibody.

We next found that the FCS concentration in the biofilm growing medium affected biofilm formation of $H$. pylori TK1402 (Fig. 4A). The lower concentrations of FCS (3.5\%, 
Table I: Optical densities and CFU measurements in the strain TKI 402 biofilm cells and broth cultures

\begin{tabular}{llll}
\hline & OD $_{600}$ & CFU $\left(\times 10^{9}\right)$ & CFU/OD $_{600}\left(\times 10^{9}\right)$ \\
\hline 2-Day biofilma & $0.141 \pm 0.037$ & $0.259 \pm 0.202$ & $1.860 \pm 1.487$ \\
3-Day biofilma & $0.258 \pm 0.027$ & $0.340 \pm 0.230$ & $1.614 \pm 0.695$ \\
2-Day broth cultureb & $0.939 \pm 0.012$ & $1.847 \pm 0.318$ & $1.966 \pm 0.387$ \\
3-Day broth cultureb & $1.075 \pm 0.044$ & $2.248 \pm 1.170$ & $2.151 \pm 1.180$ \\
\hline
\end{tabular}

\footnotetext{
a) the biofilm cells were scrapped by mechanical treatment into PBS. The cell suspensions were examined by optical densities and CFU were also calculated.

b) the standard broth cultures of strain TKI402 in Brucella broth supplemented with 7\% FCS were examined by optical densities and CFU.

Data are shown as the mean value of the 3 -independent experiments \pm standard deviations.
}

$1.75 \%$ or 0 ) caused a significant decrease in biofilm formation in this strain compared to that in $7 \%$ FCS. Therefore, the intensity of biofilm formation was dependent upon the concentration of FCS. The OMV were isolated from the cells under these conditions and characterized by SDS-PAGE (Fig. 4B). As the components of FCS might be present in the OMV fraction, the control fractions from Brucella broth supplemented with various concentration of FCS $(7 \%, 3.5 \% 1.75 \%$ and 0$)$ without the microorganism were used as controls. There were many protein bands which did not conform to FCS components (Fig. 4, lanes 1 to 4 vs. lanes 5 to 8 ). To quantify the production of OMV under these conditions, the OMV-fractions were analyzed by Western blotting with anti-H. pylori strain NCTC 11638 antibody. There were many positive bands and the intensity of these bands correlated with the FCS concentrations (Fig. 4C). As a negative control, control fractions from Brucella broth supplemented with $7 \%$ FCS without the microorganism were used and there were no detectable corresponding bands (Fig. 4C, lane 5). In addition, we observed the biofilms under these conditions with SEM (Fig. 4D to 4G). There were no OMV in the biofilms of Brucella medium only (Fig. 4D). In contrast, a large number of the OMV were detected in biofilms in Brucella broth supplemented with 7\% FCS (Fig. 4G). Under these conditions, the quantity of the OMV in the biofilm appeared to be dependent upon the concentration of FCS (Fig. 4D to $4 \mathrm{G}$ ). These results suggested that the production of OMV might be related to the biofilm forming ability of strain TK1402.

We further determined that 3-day biofilm formation with strain TK1402 in Brucella broth supplemented with 7\% HS or $0.2 \% \beta$-cyclodextrin was significantly weaker than that in Brucella broth supplemented with 7\% FCS (Fig. $5 \mathrm{~A})$. Other strains, which form thin biofilms in Brucella broth supplemented with 7\% FCS, also formed weaker biofilms, similar to or weaker than those in FCS broth with either horse serum or $\beta$-cyclodextrin. The final densities of strain TK1402 evaluated by $\mathrm{OD}_{600}$ units after 3 days of culture were $0.96 \pm 0.09,1.11 \pm 0.19$, and $0.87 \pm 0.13$ following growth with Brucella broth supplemented with $7 \%$ FCS, $7 \%$ HS, or $0.2 \% \beta$-cyclodextrin, respectively. We then isolated the OMV from TK1402 cultured in Brucella broth containing $7 \%$ FCS, $7 \%$ HS, or $0.2 \% \beta$-cyclodextrin and Western blotting with the anti-H. pylori antibody was carried out (Fig. 5C). The 50- to $60-\mathrm{kDa}$ OMV protein band intensities from growth in Brucella broth supplemented with $7 \%$ FCS were much greater than comparable fractions from $7 \%$ HS or $0.2 \% \beta$-cyclodextrin-grown cultures. These results suggested that lower production of OMV might lead to weaker biofilm formation in Brucella broth supplemented with $7 \%$ HS or $0.2 \% \beta$-cyclodextrin.

To directly verify that the OMV were components of the TK1402 biofilm matrix and that the production of the OMV can induce strong biofilm formation, TK1402 biofilm formation with $0.2 \% \beta$-cyclodextrin medium was analyzed following the addition of the OMV fraction from TK1402 cultures in Brucella broth containing 7\% FCS. The protein concentration of the OMV-fraction was adjusted to $2.0 \mathrm{mg} / \mathrm{ml}$ or $1.0 \mathrm{mg} / \mathrm{ml}$. The OMV fraction (total amounts were $0.2 \mathrm{mg}$ or $0.1 \mathrm{mg}$, respectively) was added to Brucella broth with $0.2 \% \beta$-cyclodextrin and biofilm formation with strain TK1402 was carried out. As the components of FCS might be present in the OMV fraction and could affect biofilm formation, a control fraction from Brucella broth supplemented with 7\% FCS without the microorganism was used. The levels of biofilm formation in the $0.2 \% \beta$-cyclodextrin medium supplemented with the control OMV fraction was similar to that of the $0.2 \% \beta$-cyclodextrin medium alone (Fig. $5 \mathrm{~B}$, lane $\beta$-cyclodextrin-control). On the other hand, the addition of the $0.1 \mathrm{mg}$ OMV fraction from TK1402 showed significantly higher levels of biofilm formation than those in $0.2 \% \beta$ cyclodextrin medium with the control fraction (Fig. 4B, $\beta$ cyclodextrin-FCS OMV 0.1). The levels of biofilm formation with OMV addition were similar to that in Brucella broth supplemented with 7\% FCS (Fig. 4B. $\beta$-cyclodextrin-FCS OMV 0.2). We further determined that the 0.1 mg OMV fraction from $H$. pylori cultured in Brucella broth containing $0.2 \% \beta$-cyclodextrin could also enhance biofilm formation but at levels lower than $0.2 \mathrm{mg}$ of this fraction. The OMV fraction induced more biofilm formation than $0.1 \mathrm{mg}$ of the OMV fraction from $7 \%$ FCS medium (Fig. 5B, $\beta$-cyclodextrin- $\beta$-cyclo OMV 0.1). 
(A)

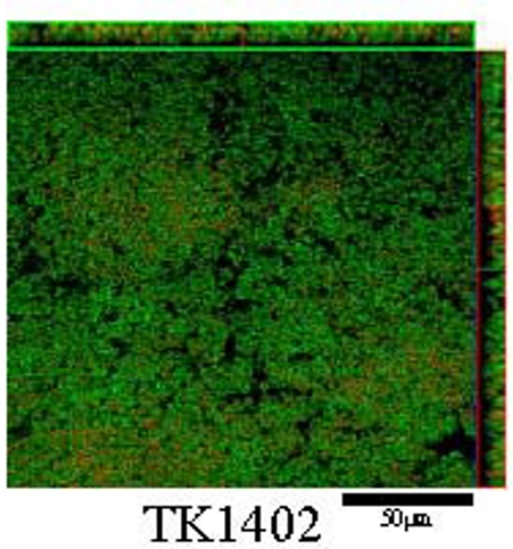

(B)

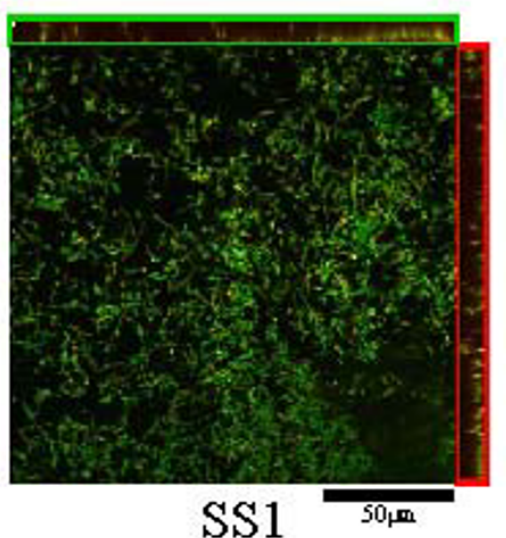

(C)

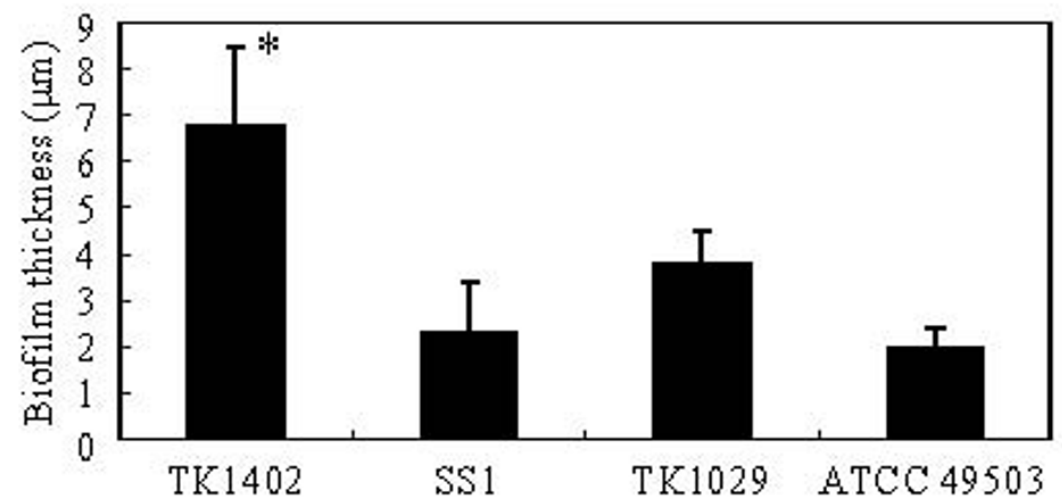

(D)

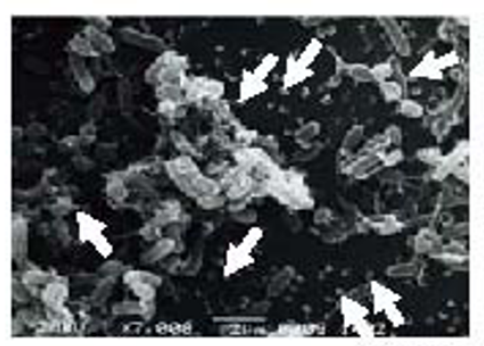

TK1402 $\times 7,000$
(E)

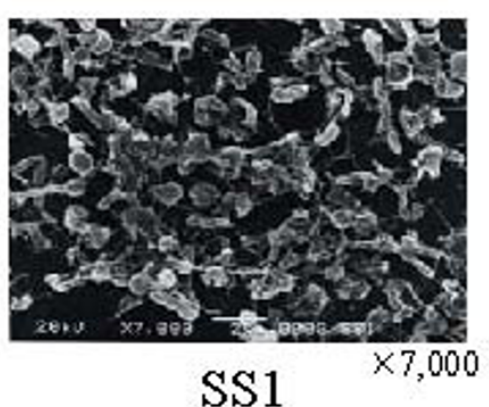

Figure 2

CLSM images of $H$. pylori strains TKI402 (A) and SSI (B) biofilms in Brucella broth containing $7 \%$ FCS. Each image represents the layer in the Z-stack that has the maximum bacterial coverage. The 3-day biofilms of each strain were stained with BacLight LIVE/DEAD stain. Viable cells are colored green and nonviable cells are colored red. $x-z$ and $y-z$ reconstructions of each biofilm are shown on the right and upper sides of each $x-y$ image. The scale bars equal $50 \mu \mathrm{m}$ in both panels. (C) Biofilm thickness of each strain. Each biofilm was scanned with CLSM at five randomly selected positions and $x$-z color detection, corresponding to biofilm thickness, was determined throughout the height of the biofilm. Data are representative of three independent experiments. The results are expressed as the means \pm standard deviations. SEM images of $H$. pylori strains TKI402 (D) and SSI (E) biofilms in Brucella broth containing 7\% FCS. The 3-day biofilm of each strain on cover glass was investigated using SEM. The OMV-like structures are indicated by white arrows (D). Scale bars $(2 \mu \mathrm{m})$ are shown at the bottom of each electron micrograph. *significantly different relative levels of biofilm thickness $(p<0.05$; strain TKI402 versus other strains). 


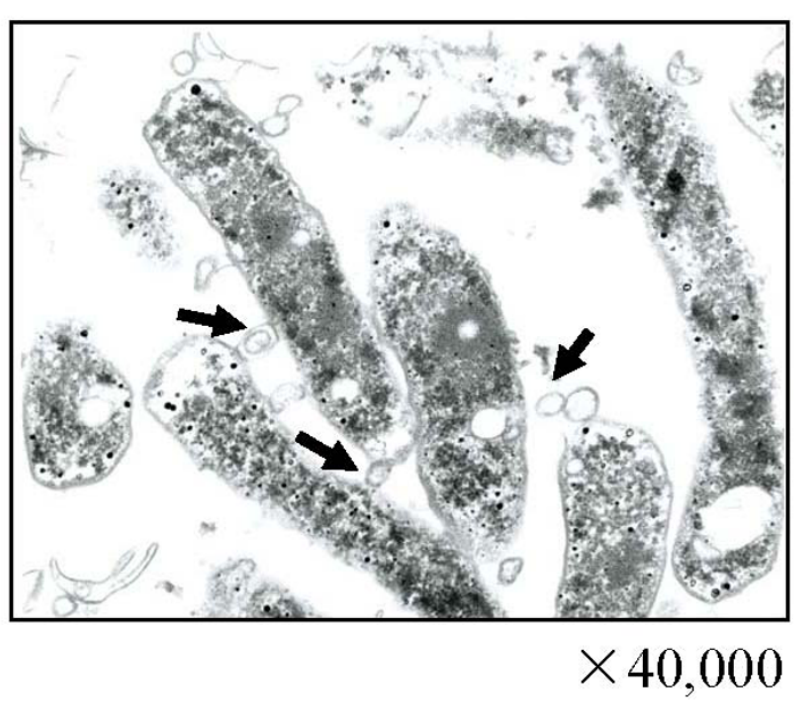

Figure 3

TEM images of $H$. pylori strain TKI 402 biofilms in Brucella broth supplemented with $7 \%$ FCS. The 3-day biofilm of strain TKI 402 on glass slides was investigated by using TEM.

\section{Evaluation of biofilm formation by other isolated $H$. Pylori strains}

In order to detect other strains having similar biofilm forming ability to strain TK1402, we assessed the biofilm forming ability of ten additional clinical isolates of $H$. pylori. Only strain TK1049 showed similar levels of biofilm formation to that of strain TK1402 (Fig. 6A). The other strains showed lower levels of biofilm formation than strain TK1402 (the biofilm $\mathrm{OD}_{595}$ values ranged from 0.1 to 0.3 ). The structure of TK1049 biofilms was then observed by using SEM (Fig. 6C). Cellular aggregation was observed to be similar to that of TK1402 biofilms and many vesicle-like structures were also detected with TK1409. Moreover, 3-day biofilm formation with strain TK1049 in Brucella broth supplemented with $0.2 \% \beta$ cyclodextrin was weaker than that in Brucella broth supplemented with $7 \%$ FCS. However, the addition of the OMV fraction from TK1402 in Brucella broth supplemented with $0.2 \% \beta$-cyclodextrin restored biofilm formation similar to that in Brucella broth supplemented with 7\% FCS (Fig. 6B).

\section{Discussion}

In this study, we characterized biofilm formation in $H$. pylori strains and demonstrated differential abilities to form biofilms in reference and clinical isolates. In addition, the production of OMV in strain TK1402 was correlated with strong biofilm formation. Several reports indicated that $H$. pylori has the ability to form biofilms on abiotic surfaces in vitro as well as on human gastric mucosa [18-21,23]. The results of the biofilm formation analyses demonstrated that strain TK1402 has strong biofilm forming ability compared to other strains independent of its growth rate. Development of strain TK1402 and SS1 biofilms from day 1 to day 6 demonstrated that it took 3 days for biofilm maturation under these conditions, suggesting that $H$. pylori biofilm formation might proceed in an organized fashion through early (Day 1), intermediate (Day 2) and maturation (after Day 3) phases of development. Similar distinct developmental phases have been reported for biofilm formation by other bacterial species [24,25]. Since development of biofilms is closely associated with the generation of a matrix, the majority of which is extracellular material, biofilm development in $H$. pylori appears to share common basic steps with other biofilm forming bacteria.

The biofilm forming cells at day 3 generally appeared to be viable when the cells were exposed to Live/Dead BacLight staining. In addition, the normalized CFU values for the biofilm and broth culture cells following 2 days of incubation were comparable. In 3-day biofilm cells, this value was slightly decreased compared to 3-day broth culture cells, suggesting the presence of some dead cells in the biofilm. These results are consistent with the maturation phase of the development of biofilms in 3-day biofilms of strain TK1402, since biofilms are thought to be encased in an EPS matrix as well as dead cells [26]. In addition, strain TK1402 exhibited thick biofilm formation. The biofilm morphology of strain TK1402 showed direct cell-cell bound aggregates as well as flagelladependent binding forms. The cell-cell interacting forms might act as precursors for thick biofilm formation. Gots et al. indicated that cell-cell aggregation induces a multilayered architecture during Staphylococcus epidermidis biofilm formation [27]. Moreover, in our SEM observations, for the majority of the H. pylori strains examined, ie., SS1, biofilms may contain autolysed cells. On the other hand, there were clearly intact cells in TK1402, as well as TK1049, biofilms and the later is also another strong biofilm forming strain. These observations suggested that these strong biofilm forming strains may remain in an active metabolic state for a relatively long time without exhibiting morphological changes or autolysis, in comparison with the other strains. These later properties could be responsible for the weaker biofilm forming activities of most of the strains examined in this study.

In the SEM observations of TK1402 biofilms, there were many OMV. OMV production is a physiologically normal function of gram-negative bacteria [22,28]. It was also reported that the $H$. pylori strains released OMV into the extracellular space $[29,30]$. A recent report indicated that OMV were a component of the matrix of $P$. aeruginosa bio- 


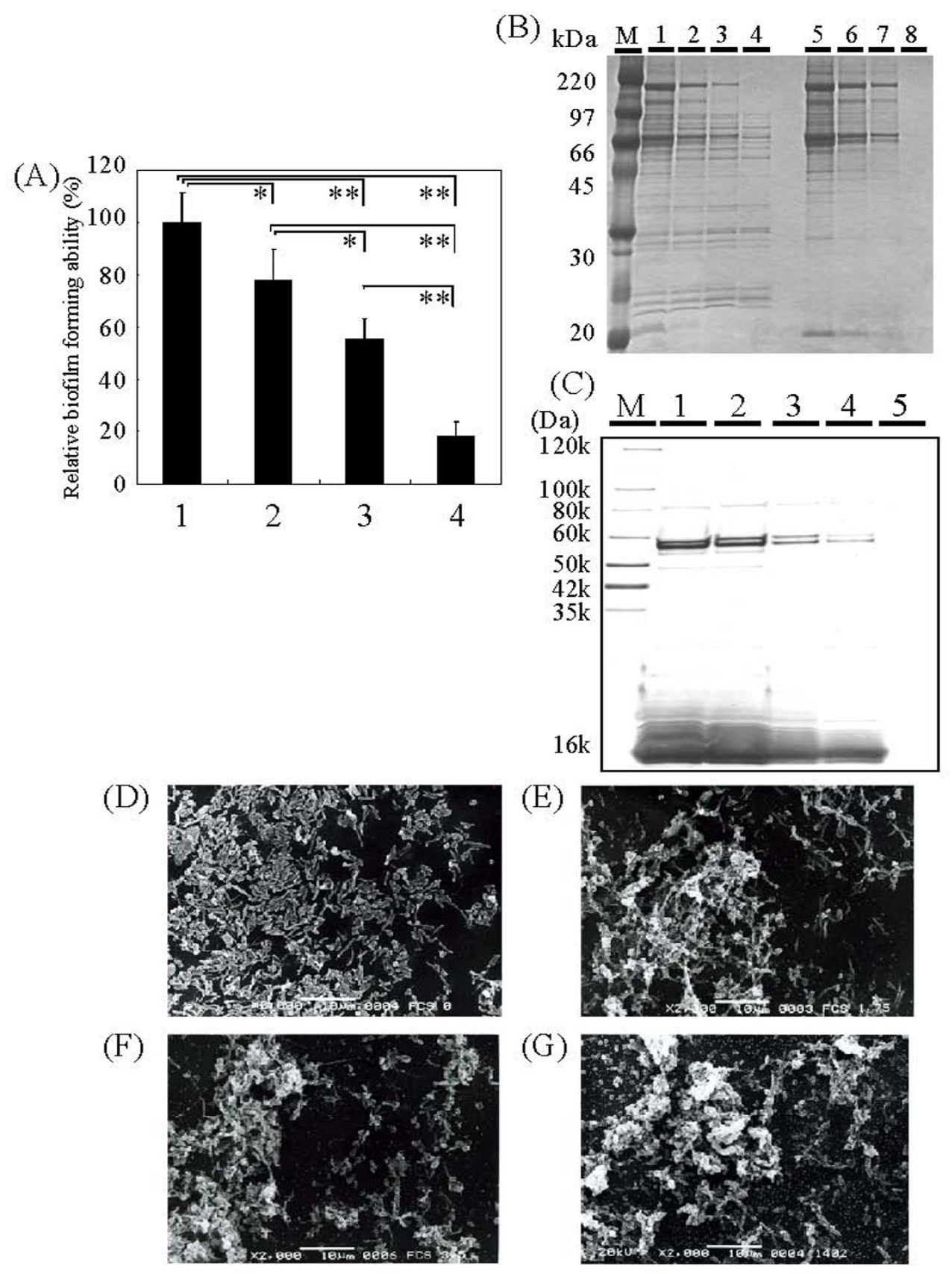

Figure 4

(A) Effects of FCS concentrations in the biofilm growth medium on TKI 402 biofilm formation. Strain TKI402 biofilms in Brucella broth supplemented with various concentrations of FCS (7\%: lane I, 3.5\%: lane 2, I.75\%: lane 3 and 0: lane 4) were examined. Quantification of biofilms (percent) was calculated relative to that of strain TKI 402 in Brucella broth supplemented with $7 \%$ FCS, which was set equal to $100 \%$. The values for the biofilms under these conditions are shown as in Fig. IA. (B) The OMV were fractionated from different medium conditions for TKI 402 cultures and the OMV-fractions were separated by SDS-PAGE (lane I, 7\% FCS; lane 2, 3.5\%; lane 3, I.75\% lane 4, Brucella broth only) and compared to controls (medium without the organism, FCS concentrations were 7\%: lane 5, 3.5\%: lane 6, I.75\%: lane 7 and 0: lane 8). (C) Western blotting of OMV-fraction from different medium conditions using anti-H. pylori antibody. M: Molecular weight marker. Lanes: I, 7\% FCS; 2, 3.5\%; 3, I.75\%; 4, 0; 5, 7\% FCS without organism (negative control). (D to G) SEM observation of TKI402 biofilms under different medium conditions. D: Brucella broth only (without FCS, 0); E: with I.75\% FCS; F: with 3.5\% FCS; G: with 7\% FCS. *significantly different $(p<0.05)$. ** significantly different $(p<0.005)$. 

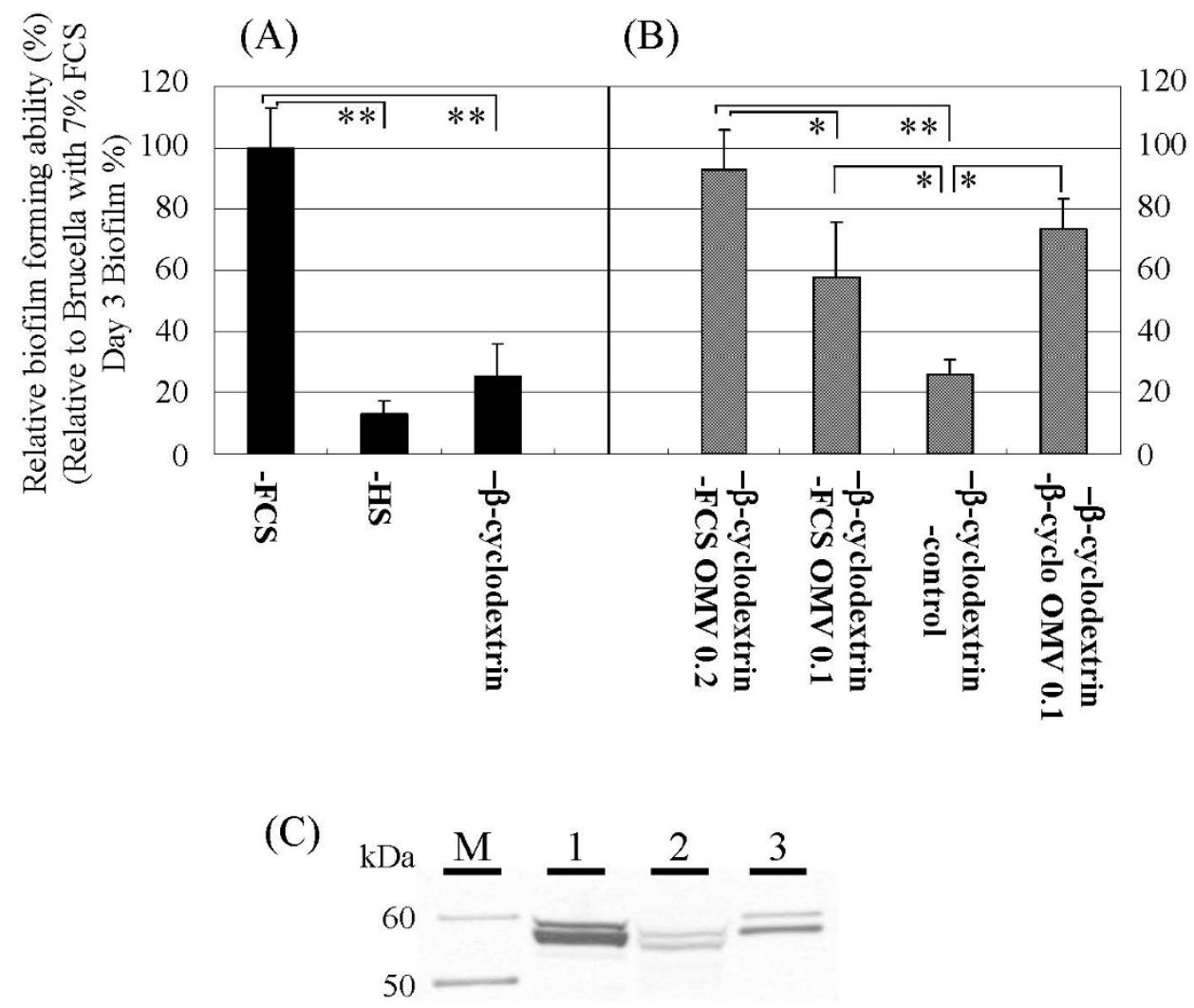

\section{Figure 5}

(A) Biofilm formation by strain TKI 402 in Brucella broth supplemented with 7\% FCS (-FCS), 7\% HS (-HS), or with $\mathbf{0 . 2} \% \beta$-cyclodextrin (- $\beta$-cyclodextrin). Relative biofilm forming activity (percent) was calculated relative to the 3 -day biofilm in Brucella broth supplemented with 7\% FCS. Data are expressed as the means of all of experiments \pm standard deviations. (B) The OMV-fraction was added to Brucella broth supplemented with $\beta$-cyclodextrin. The protein concentrations in the OMV-fractions were adjusted and $0.2 \mathrm{mg}$ of the OMV-fraction ( $\beta$-cyclodextrin-FCS OMV 0.2 ), or $0.1 \mathrm{mg}$ of the OMV-fraction ( $\beta$-cyclodextrin-FCS OMV 0.I) were added. Control fractions from the medium without bacteria were also added ( $\beta$-cyclodextrin-control). Further, the OMV-fraction was isolated from this organism in Brucella broth supplemented with $0.2 \% \beta$ cyclodextrin and $0.1 \mathrm{mg}$ of the OMV-fraction from $0.2 \% \beta$-cyclodextrin medium was added ( $\beta$-cyclodextrin- $\beta$-cyclo OMV 0.1 ). Biofilm formation was examined after 3 days of culture. Relative biofilm forming activity (percent) was calculated relative to the 3-day biofilm in Brucella broth supplemented with 7\% FCS. Data are expressed as the means of all of experiments \pm standard deviations. (C) Western blotting of the OMV-fraction from different medium conditions using anti-H. pylori antibody. M: Molecular weight marker. Lanes: I, 7\% FCS; $2,7 \%$ HS; 3, 0.2\% $\beta$-cyclodextrin. *significantly different $(p<0.05)$. ** significantly different $(p<0.005)$.

films [14]. We analyzed thin-sections of strain TK1402 biofilms with TEM. The OMV were located at the substratum-bacterium interface and extracellular space. Interestingly, some of OMV appeared to be involved in attaching one cell with another. This observation suggested that the OMV produced by strain TK1402 could be intimately involved in biofilm formation.

Previously, several reports indicated that VacA, urease and lipopolysaccharides are present on the surface of OMV from $H$. pylori along with other outer membrane proteins $[29,30]$. We quantified OMV production in Brucella broth supplemented with various concentration of FCS using Western blotting with anti-H. pylori antibody. Moreover, the SEM observations were also carried out to directly confirm this. The FCS concentration in the biofilm medium showed a direct positive correlation with OMV production as well as biofilm forming ability. Further, similar results were detected by the addition of serum from different hosts as well as with synthetic substrates. On the other hand, observation with biofilm forming bacteria indicated that LPS plays a role in biofilm development and architecture [14,31]. Recently, Keenan et al. reported that LPS detected in OMV under iron-limited conditions were 
(A)

(B)

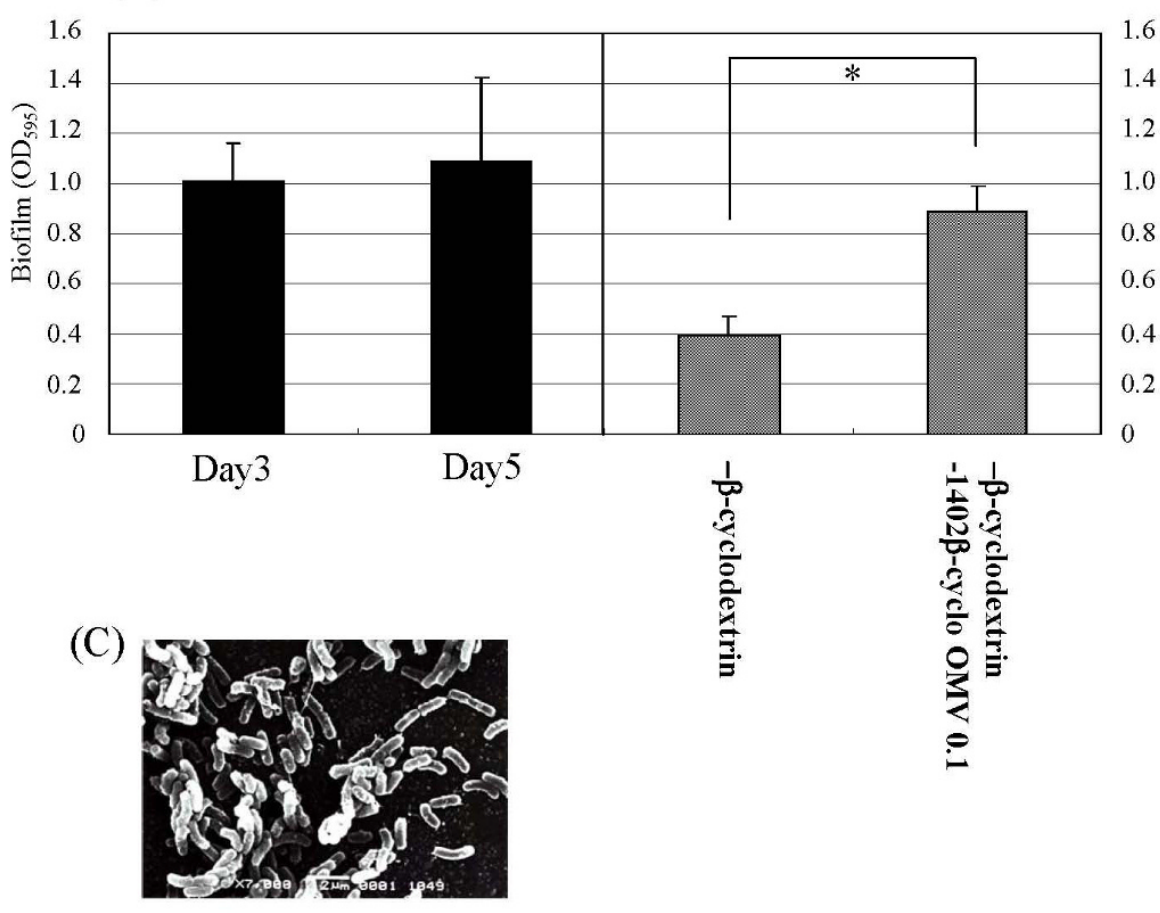

Figure 6

(A) Biofilm formation by strain TK 1049. Graph shows quantification of biofilms formed after 3-day (Day 3) and 5-days (Day 5) in Brucella broth supplemented with 7\% FCS. (B) Biofilm formation by strain TK 049 in Brucella broth supplemented with $0.2 \% \beta$-cyclodextrin and addition of the OMV-fraction from TKI 402 grown in $0.2 \% \beta$-cyclodextrin medium. (C) SEM observation of TK 1049 biofilms. *significantly different $(p<0.05)$.

notably shorter than those under iron-replete conditions [32]. We hypothesize that strain TK1402 has an altered LPS, particularly LPS O-antigens under different experimental conditions including the use of different animal sera, synthetic substrates, or different FCS concentrations. To confirm this, we analyzed the LPS profiles of $H$. pylori cultured in different culture media by SDS-PAGE and silver staining. However, there were no differences in the LPS O-antigen profiles. We then isolated the OMV from the TK1402 culture supernatant in order to examine the role of these structures in biofilm formation. Biofilm formation by this strain was increased following the addition of the OMV-fraction in a dose-dependent manner. Although the quantities of OMV added were three- to fivefold more than the quantity of the OMV which exist in biofilms under our experimental conditions, the OMV appear to play an important role in the formation of the extracellular matrix of strain TK1402 biofilms. The extracellular matrix serves a role in bacterial attachment to abiotic and cellular surfaces in the initial stage of biofilm formation [33]. It is possible that specific proteins in the OMV released from strain TK1402 may take part in bacterial aggregation and biofilm formation. Which compo- nent(s) of the OMV contribute to biofilm formation still remains to be determined. Additional investigations are now in progress to determine such components in the OMV.

In the present study, we searched for other clinical isolates with strong biofilm formation and one strain, TK1049, exhibited similar ability to form biofilms as strain TK1402. This suggested that $H$. pylori strains exhibiting strong biofilm forming ability are relatively rare. Indeed, Williams et al. indicated that FCS inhibited adherence to abiotic surfaces in some of the H. pylori strains [34]. This apparent discrepancy between their study and our present results in terms of the effects of FCS might be due to differences in the H. pylori strains used.

Strain TK1402 was isolated from a patient with duodenal and gastric ulcers in Japan. This strain contains the cagA, cagPAI and vacA genes as demonstrated by PCR [35]. It was also shown that this strain expresses the Lewis ${ }^{y}$ antigen (LeY) on the cell surface. Moreover, strain TK1402 was reported to exhibit virulence in gnotobiotic mice [36], C57BL mice [37], and Mongolian gerbils [35]. These 
reports indicated that the TK1402 strain has the ability to colonize the stomach of these animals as well as in humans. These results as well as our present findings suggest that this colonization ability might be correlated with the strong biofilm forming ability of strain TK1402. Therefore, we speculate that strong biofilm forming ability is related to gastric colonization by $H$. pylori in various animals as well as in humans. It is recognized that an understanding of $H$. pylori biofilm formation is still in its infancy. The ability of $H$. pylori strains, as exemplified by strain TK1402, to form biofilms may play a part of role in the infectious process.

\section{Conclusion}

We have demonstrated that strain TK1402 has strong biofilm forming ability. In addition, the results suggested that this property is dependent upon direct cell-cell binding mediated by the OMV of this strain. This represents a new observation relative to a potentially novel gastric cell colonization factor of this organism.

\section{Methods}

\section{Bacterial strains and culture conditions}

The following $H$. pylori strains were used: SS1, ATCC 49503, ATCC 43579, NCTC11638, TK1029, TK1402, KR2003, and KR2005. The last four are clinical isolates from Japanese patients. Strains TK1029 and TK1402 were used as described previously [38]. In addition, strains TK1036, TK1042, TK1043, TK1045, TK1046, TK1047, TK1049, TK1054, TK1056, and TK1057 were also used for assessing biofilm forming ability. Strains KR2003 and KR2005, as well as the latter strains were isolated from a gastritis patient in our laboratory. All strains were maintained at $-80^{\circ} \mathrm{C}$ in Brucella broth (Difco, Detroit, Mich) with $20 \%$ ( vol/vol) glycerol. These strains were cultured under microaerobic conditions at $37^{\circ} \mathrm{C}$ on Brucella agar plates containing $7 \%$ horse serum (HS).

\section{Biofilm formation and its quantification}

Biofilm formation by all strains was carried out as previously described $[19,20]$ with slight modifications. Briefly, sterilized glass coverslips (approximately $22 \times 22-\mathrm{mm}$, 0.12 to $0.17-\mathrm{mm}$ thickness, Matsunami Glass, Tokyo, Japan) were placed into 12-well microtiter plates. Each well was filled with $2 \mathrm{ml}$ of Brucella broth supplemented with $7 \%$ fetal calf serum (FCS), $7 \%$ horse serum (HS), or $0.2 \% \beta$-cyclodextrin to allow adherence of $H$. pylori at the air-liquid interface. The formation of biofilms was initiated by inoculating $10 \mu \mathrm{l}$ of pre-cultured cell suspension (approximately $5 \times 10^{5}$ cells in Brucella broth) into each well. The cultures were incubated under microaerobic conditions at $37^{\circ} \mathrm{C}$ for 1 to 6 days with shaking (80-100 $\mathrm{rpm})$. After incubation, the coverslips were removed and washed with phosphate-buffered saline (PBS). The samples were then air dried and stained with crystal violet for $30 \mathrm{~s}$. After being stained, the coverslips were rinsed with distilled $\mathrm{H}_{2} \mathrm{O}$ to remove excess dye and then air dried for $30 \mathrm{~min}$. All dye associated with the biofilms was dissolved with $1 \mathrm{ml}$ of ethanol and $200 \mu \mathrm{l}$ of the ethanol solutions were used to measure the absorbance at $594 \mathrm{~nm}$ with a microplate reader to determine the amount of biofilm formation.

\section{Confocal laser scanning microscopy (CLSM) and measurement of biofilm thickness}

For visualization, the biofilms of $H$. pylori strains on the coverslips were stained with a BacLight LIVE/DEAD bacterial viability kit solution (Molecular Probes, Leiden, The Netherlands) according to the directions of the supplier. Confocal images were collected by using a Zeiss LSM 510META confocal laser scanning microscope (Carl Zeiss, Jena, Germany). To determine biofilm thickness, a series of horizontal $(x z)$ optical sections at $0.5 \mu \mathrm{m}$ intervals were taken through the height of the biofilm for measurement. Each biofilm was scanned at five randomly selected positions. Each sample was observed independently more than three times. Confocal images of green and red fluorescence were constructed simultaneously using a multitrack mode.

\section{Cell viability assay}

To determine the numbers of viable bacteria, biofilm cells on the coverslips were scrapped into PBS. The optical densities and colony-forming units (CFU) of the cell suspensions were quantitated as the mean of three independent observations. As controls, standard cell broth cultures were used.

\section{Electron microscopic studies}

To observe the biofilm ultrastructure, the biofilms formed on the coverslips were examined by scanning electron microscopy (SEM). The biofilms on the coverslips were fixed with $2 \%$ glutaraldehyde for $3 \mathrm{~h}$ at room temperature and the samples were observed using a JSM-5600LV electron microscope (JEOL, Tokyo, Japan).

To observe the OMV-like structures, the biofilms of strain TK1402 on the glass slides were examined by using transmission electron microscopy (TEM). Glass slides cut in half were placed into 6-well microtiter plates and the biofilms were allowed to form as described above. The biofilms were fixed with $2 \%$ glutaraldehyde for $3 \mathrm{~h}$ at room temperature. The samples were then dehydrated and embedded in Epon 813 embedding solution (Chemische Werke Lowi GmbH, Waldkaraigurg, Germany). The sections were finally observed with a JEM-100 electron microscope (Jeol).

\section{Isolation of outer membrane vesicles}

Isolation of OMV was performed as described previously [30]. Briefly, H. pylori strain TK1402 was grown in Brucella broth supplemented with various concentration of FCS 
for 3 days. After cultivation, the optical density at $600 \mathrm{~nm}$ of the cell cultures was adjusted to 0.5 with each respective medium. The cells were collected by centrifugation $(10,000 \mathrm{~g}$ for $15 \mathrm{~min})$, and the resulting supernatants were filtered (low protein binding Durapore membrane, $0.45 \mathrm{~mm}$ polyvinylidene fluoride, Millipore, Bedford, Mass.). The filtrates were centrifuged $(40,000 \mathrm{~g}, 2 \mathrm{~h}$ at $\left.4^{\circ} \mathrm{C}\right)$, washed with PBS and re-centrifuged $(40,000 \mathrm{~g}, 2 \mathrm{~h}$ at $4^{\circ} \mathrm{C}$ ). The pellets were next resuspended in PBS supplemented with $0.2 \mathrm{M} \mathrm{NaCl}$. The media without the bacteria were used as controls. The OMV of strain TK1402 in Brucella broth supplemented with $0.2 \% \beta$-cyclodextrin or $7 \%$ horse serum were also isolated in a similar manner.

\section{Sodium dodecyl sulfate-polyacrylamide gel electrophoresis (SDS-PAGE) and immunoblotting techniques}

The fractionated OMV (OMV-fraction) were treated with sodium dodecyl sulfate (SDS) loading buffer including $5 \%$ 2-mercaptoethanol at $100^{\circ} \mathrm{C}$ for $5 \mathrm{~min}$ and separated by polyacrylamide gel electrophoresis (PAGE). The separated OMV proteins were stained with Coomassie brilliant blue. For Western blotting assays, the OMV-fractions were loaded onto gels and transferred to polyvinylidene difluoride membranes (Atto, Tokyo, Japan). After transfer, the membranes were blocked with $3 \%$ bovine serum albumin in PBS for $60 \mathrm{~min}$ and incubated with $H$. pylori strain NCTC 11638 whole-cell antiserum $(1: 2,000)$ [36] for 60 min. After washing with PBS containing 0.05\% Tween 20 (PBST), peroxidase-labeled goat anti-rabbit immunogloblins (Dako A/S, Glostrup, Denmark) were used at 1:2,000 dilution as secondary antibodies. After washing with PBST, the blots were developed.

\section{Complementation of biofilm forming ability using the OMV}

The OMV-fraction from Brucella broth supplemented with 7\% FCS (OMV-fraction) and the medium fraction (control-fraction) in PBS were adjusted to an optical density of 2.0, or 1.0 at $280 \mathrm{~nm}$. The OMV-fractions from Brucella broth supplemented with $0.2 \% \beta$-cyclodextrin were also adjusted to optical densities of 1.0. After filtration, $100 \mu \mathrm{l}$ of the fractionated OMV were added to Brucella broth with $0.2 \% \beta$-cyclodextrin for TK1402 biofilm formation assays (described above).

\section{Statistical analysis}

Statistical analysis was performed using the Mann-Whitney $U$ test. P values of 0.05 or less were considered to indicate statistical significance.

\section{Authors' contributions}

HY carried out the experiments and drafted the manuscript. TO contributed to the experimental concept and design as well as provision of technical support. SKu initially conceived the idea for this study. MF carried out the microscopy techniques while HK and KO participated in discussions regarding the study design. TH contributed to the experimental concept and design as well as assisting in technical support. SKa was also involved in the conception of this study, and participated in its design and coordination as well as helping to draft the manuscript. All authors read and approved the final manuscript.

\section{Acknowledgements}

This work was supported by Grants for Scientific Research 18590437 from the Ministry of Education, Culture, Sport, Science and Technology and a grant from the Dental Research Center, Nihon University School of Dentistry.

\section{References}

I. Marshall BJ, Warren JR: Unidentified curved bacilli in the stomach of patients with gastritis and peptic ulceration. Lancet 1984, I6:13|I-|3|5.

2. Blaser MJ: Helicobacter pylori: its role in disease. Clin Infect Dis 1992, 15:386-39|.

3. Graham DY: Campylobacter pylori and peptic ulcer disease. Gastroenterology 1989, 96:615-625.

4. Parsonne J, Friedman GD, Vandersteen DP, Chang Y, Vogelman JH, Orentreich N: Helicobacter pylori infection and the risk of gastric carcinoma. N Engl J Med I99|, 325:| |27-I |3|.

5. Wotherspoon AC, Doglioni C, Diss TC, Pan L, Moschini A, De Boni $M$, Isaacson PG: Regression of primary lowgrade B-cell gastric lymphoma of mucosa-associated lymphoid tissue type after eradication of Helicobacter pylori. Lancet 1993, 342:575-577.

6. Akada JK, Shirai M, Takeuchi H, Tsuda M, Nakazawa T: Identification of the urease operon in Helicobacter pylori and its control by mRNA decay in response to pH. Mol Microbiol 2000, 36: $1071-1084$.

7. Bijlsma J], Vandenbroucke-Grauls CM, Phadnis SH, Kusters JG: Identification of virulence genes of Helicobacter pylori by random insertion mutagenesis. Infect Immun 1999, 67:2433-2440.

8. Censini S, Lange C, Xiang Z, Crabtree JE, Ghiara P, Borodovsky M, Rappuoli $R$, Covacci A: cag, a pathogenicity island of Helicobacter pylori, encodes type I-specific and disease-associated virulence factors. Proc Natl Acad Sci USA 1996, I 0: | 4648- 4653.

9. Hall-Stoodley L, Costerton JW, Stoodley P: Bacterial biofilm: from the nature environment to infectious diseases. Nat Rev Microbiol 2004, 2:95-108.

10. Burne RA: Oral streptococci; products of their environment. J Denat Res 1998, 77:445-452.

II. Danes PN, Pratt LA, Kolter R: Exopolysaccharide production is required for development of Escherichia coli K-12 biofilm architecture. J Bacteriol 2000, 182:3593-3596.

12. Fux CA, Costerton JW, Stewart PS, Stoodley P: Survival strategies of infectious biofilms. Trend Microbiol 2005, 13:34-40.

13. Rupp ME, Fey PD, Heilmann C, Gotz F: Characterization of the importance of Staphylococcus epidermidis autolysin and polysaccharide intercellular adhesion in the pathogenesis of intravascular catheter-associated infection in a rat model. J Infect Dis 2001, 183:1038-1042.

14. Schooling SR, Beveridge TJ: Membrane vesicles: an overlooked component of the matrices of biofilms. J Bacteriol 2006, 188:5945-57.

15. Costerton JW, Lewandowski Z, Caldwell DE, Korber DR, LappinScott HM: Microbial biofilms. Annu Rev Microbiol 1995, 49:7 I I-745.

16. Sutherland IW: The biofilm matrix-an immobilized but dynamic microbial environment. Trends Microbiol 200I, 9:222-227.

17. Mackay WG, Gribbon LT, Barer MR, Reid DC: Biofilms in drinking water systems-a possible reservoir for Helicobacter pylori. Water Sc Technol 1998, 38:18I-185.

18. Stark RM, Gerwig G], Pitman RS, Potts LF, Williams NA, Greenman J, Weinzweig IP, Hirst TR, Millar MR: Biofilm formation by Helicobacter pylori. Lett Appl Microbiol 1999, 28:121-6.

19. Cellini L, Grande R, Di Campli E, Di Bartolomeo S, Di Giulio M, Traini T, Trubiani O: Characterization of an Helicobacter pylori environmental strain. J Appl Microbiol 2008, 105:76I-9. 
20. Cole SP, Harwood J, Lee R, She R, Guiney DG: Characterization of monospecies biofilm formation by Helicobacter pylori. J Bacteriol 2004, I86:3124-3I32.

21. Carron MA, Tran VR, Sugawa C, Coticchia JM: Identification of Helicobacter pylori biofilms in human gastric mucosa. J Gastrointest Surg 2006, 10:7I 2-7I7.

22. Lee EY, Choi DS, Kim KP, Gho YS: Proteomics in gram-negative bacterial outer membrane vesicles. Mass Spectrom Rev 2008, 27:535-55.

23. Park SR, Mackay WG, Reid DC: Helicobacter sp. recovered from drinking water biofilm sampled from a water distribution system. Water Res 200I, 35:1624-6.

24. Davey ME, O'Toole GA: Microbial biofilms: from ecology to molecular genetics. Microbiol Mol Biol Rev 2000, 64:847-867.

25. O'Toole GA, Kaplan HB, Kolter R: Biofilm formation as microbial development. Annu Rev Microbiol 2000, 54:49-79.

26. Qin Z, Ou Y, Yang L, Zhu Y, Tolker-Nielsen T, Molin S, Qu D: Role of autolysin-mediated DNA release in biofilm formation of Staphylococcus epidermidis. Microbiology 2007, 153:2083-92.

27. Götz F, Heilmann C, Cramton SE: Molecular basis of catheter associated infections by staphylococci. Adv Exp Med Biol 2000, 485: $103-11$.

28. Beveridge T]: Structures of gram-negative cell walls and their derived membrane vesicles. J Bacteriol 1999, I $81: 4725-4733$.

29. Fiocca R, Necchi V, Sommi P, Ricci V, Telford J, Cover TL, Solcia E: Release of Helicobacter pylori vacuolating cytotoxin by both a specific secretion pathway and budding of outer membrane vesicles. Uptake of released toxin and vesicles by gastric epithelium. J Pathol 1999, 188:220-226.

30. Keenan JI, Allardyce RA, Bagshaw PF: Dual silver staining to characterize Helicobacter spp. outer membrane components. Immunol Methods 1997, 209: 17-24.

31. Danes PN, Pratt LA, Kolter R: Exopolysaccharide production is required for development of Escherichia coli K-12 biofilm architecture. J Bacteriol 2000, 182:3593-3596.

32. Keenan JI, Davis KA, Beaugie CR, McGovern JJ, Moran AP: Alterations in Helicobacter pylori outer membrane and outer membrane vesicle-associated lipopolysaccharides under ironlimiting growth conditions. Innate Immun 2008, 14:279-290.

33. Costerton JW, Cheng KJ, Geesey GG, Ladd TI, Nickel JC, Dasgupta M, Marrie TJ: Bacterial biofilms in nature and disease. Annu Rev Microbiol 1987, 4I:435-464.

34. Williams JC, Mclnnis KA, Testerman TL: Adherence of Helicobacter pylori to abiotic surfaces is influenced by serum. Appl Environ Microbiol 2008, 74: I255-1258.

35. Nakagawa S, Osaki T, Fujioka Y, Yamaguchi H, Kamiya S: Long-term infection of Mongolian gerbils with Helicobacter pylori: microbiological, histopathological, and serological analyses. Clin Diagn Lab Immunol 2005, I 2:347-353.

36. Osaki T, Taguchi H, Yamaguchi H, Kamiya S: Detection of Helicobacter pylori in fecal samples of gnotobiotic mice infected with $H$. pylori by an immunomagnetic-bead separation technique. J Clin Microbiol I 998, 36:32 I-323.

37. Yamaguchi $\mathrm{H}$, Osaki T, Taguchi H, Hanawa T, Yamamoto $\mathrm{T}$, Kamiya S: Flow cytometric analysis of the heat shock protein 60 expressed on the cell surface of Helicobacter pylori. J Med Microbiol 1996, 45:270-277.

38. Yamaguchi H, Osaki T, Taguchi H, Sato N, Toyoda A, Takahashi M, Kai M, Nakata N, Komatsu A, Atomi Y, Kamiya S: Effect of bacteria flora on postimmunization gastritis following oral vaccination of mice with Helicobacter pylori heat shock protein 60. Clin Diagn Lab Immunol 2003, 10:808-8I2.

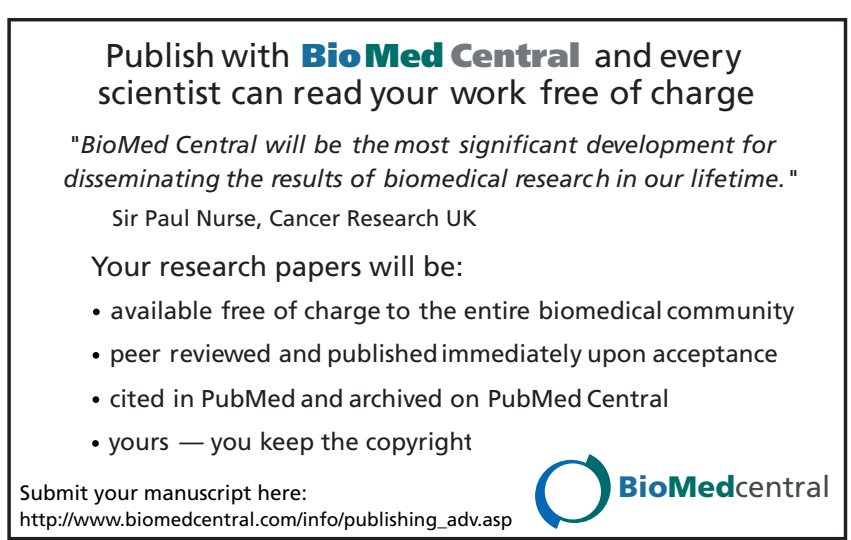

